\title{
Measurement of reed valve kinematics
}

\author{
Michael Fenkl ${ }^{1, a}$, Václav Dvořák ${ }^{1}$ and Tomáš Vit ${ }^{1}$ \\ ${ }^{1}$ Technical University of Liberec, Department of Power Engineering Equipment, Studentská 1402/2, Liberec I, Czech Republic
}

\begin{abstract}
The measurement of key kinematic parameters of a reed valve movement is necessary for the further development of the reed valve system. These parameters are dependent on the geometry and material properties of the valve. As they directly affect the quantity of air flowing around the valve, a simple and easy to implement measurement of various valve configuration based on the air flow has been devised and is described in this paper, along with its technical parameters and drawbacks when evaluating reed valves used in reciprocating air compressors. Results are presented for a specimen of a compressor under examination. All kinematic parameters, and timing of the opening and closing of the valve, obtained from the measurement are presented and discussed.
\end{abstract}

\section{Introduction}

Reed valve systems are thanks to their simplicity frequently used for regulation of suction and discharge strokes of reciprocating compressors. For a meaningful development of such systems, the kinematic parameters of the valves during a realistic operation, which depend on material properties and geometry of the system, need to be known. A suitable method for measuring the valve motion and subsequent data analysis is necessary for determination of such parameters. Given that the valvetrain is enclosed in the compressor head, any measurement requires certain modifications to the compressor. Measurement on a test stand, where the valve motion is simulated using compressed gas and the motion is recorded by a high-speed camera, is one of the simplest measurement methods [1]. This approach, however, does not reflect all effects present during realworld operation of the compressor. Another approach is a numerical solution of the problem using Computational Fluid Dynamics and Finite Elements Method (CFD/FEM) $[2,3]$. However, given the substantial complexity of the computations and quantity of the input data the interaction of a compressible fluid (gas) and a non-rigid body (valve) is greatly simplified, leading to less accurate results. Another approach is based on the use of an endoscope coupled to a high-speed camera to record a realistic reed valve movement [4], which requires, however, a rather complex data analysis. Yet another method is the examination of a combustion engine valve motion relative to the cam shape using a vibrometer [5].

\section{Methods}

\subsection{Measurement setup}

A Laser Doppler vibrometer Ometron VH-1000-D (Fig. 1), with a voltage output directly proportional to the velocity of the sensed object, was attached to the head of a tested compressor specimen using a custom-fabricated holder above a hole drilled above suction and discharge valves, fitted with a glass plate for non-contact sensing of the valve motion. The dynamic pressure in the cylinder and in the discharge duct downstream of the discharge valve was measured using a pair of optomechanical pressure sensors (Fig. 2, AutoPSI D82294-Q, Optrand, USA) (2). All three signals were synchronized with the crankshaft position sensed by an incremental encoder (Fig. 3, ARC 425/10KB) with a resolution of 512 points per revolution. All data were captured by a fast data acquisition board (Fig. 3, NI USB 6218). A Gray code to binary code converter was placed in between the incremental encoder and the data acquisition module.

The piston bottom dead center was chosen as the zero position of the incremental encoder. The output pressure was set by a needle valve capable of maintaing a constant pressure during a steady-state operation of the compressor. The control and data acquisition software working at a resolution of 512 data points per crankshaft revolution was written by the authors in LabView (National Instruments).

\footnotetext{
${ }^{\mathrm{a}}$ Corresponding author: michael.fenk1@tul.cz
} 


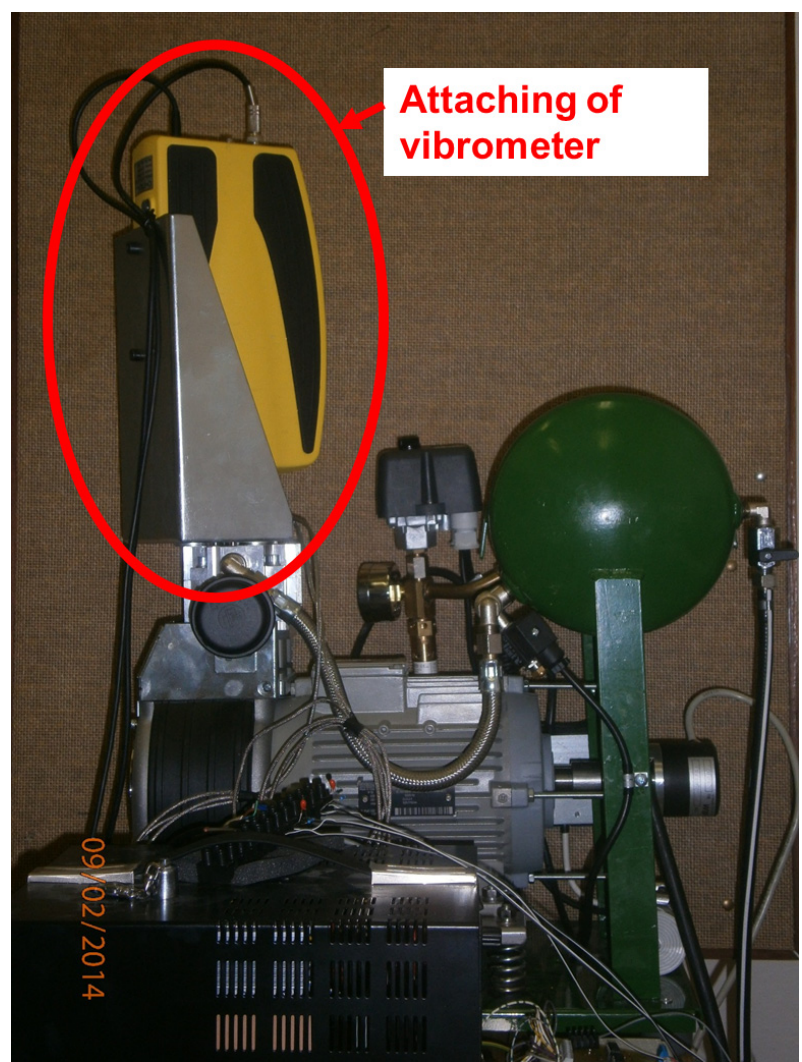

Figure 1. Vibrometer attachment.

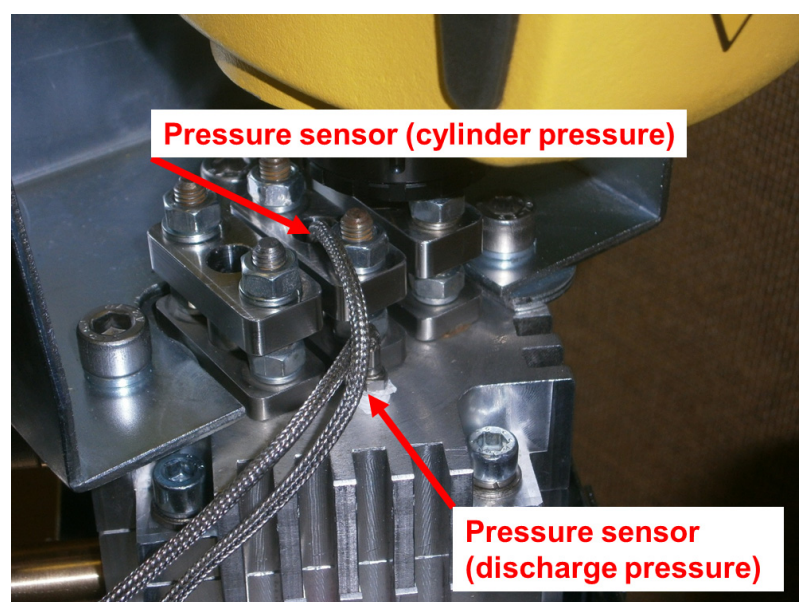

Figure 2. Installation of dynamic pressure sensors.

\subsection{Drawbacks of the measurement method}

Several minor issues were successfully resolved prior to the measurement. The own vibrations of the vibrometer, contributed to by its considerable mass, affected the measurement, which had to be compensated by subtracting vibrometer vibrations recorded during a series of measurements with the glass plate covered for each measured regime. This has greatly increased the accuracy of the measurement of the valve velocity, however, the measurement were still affected by the internal frequency of the vibrometer holder. The standard deviation of the valve velocity was up to $6.6 \%$ for the discharge valve and up to, in several cases, $20 \%$ for the suction valve affected by a considerable variability of the valve motion due to underpressure in the cylinder. During a comparable measurement of dynamic pressures in the cylinder and in the discharge duct, which was not affected by vibrations, the standard deviation did not exceed $0.2 \%$ for 10 thousands subsequent working cycles.

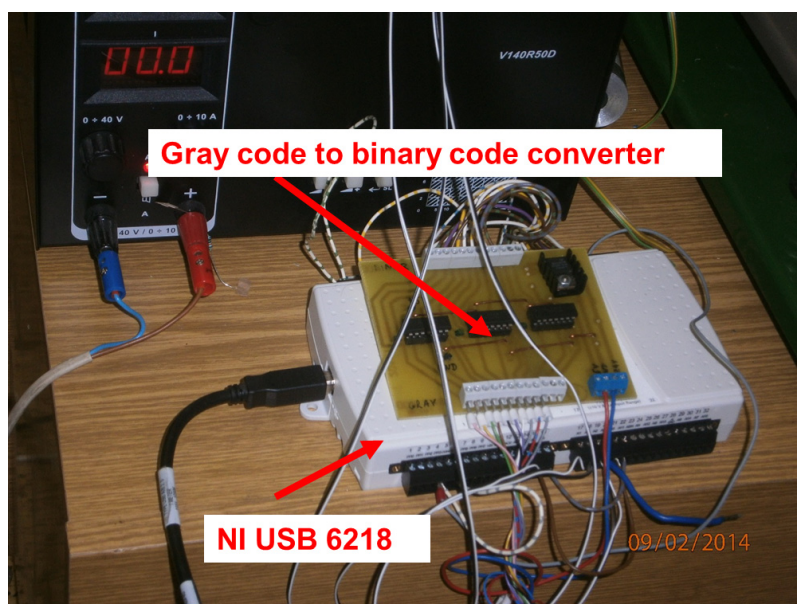

Figure 3. Data acquisition system.

\section{Results and discussion}

The measurement took place at the discharge pressure of 300,500 and $800 \mathrm{kPa}$ for four sets of valves A, B, C and D. Suction and discharge valves in sets $\mathrm{A}$ and $\mathrm{B}$ were identical except that valves $B$ were thinner. In sets $C$ and $\mathrm{D}$, the valves were identical except that valves $\mathrm{D}$ were thinner. Valves C and D were longer than A and B. Ten thousands of working cycles were recorded, at 512 data points per cycle, and mean values were computed for each of the 512 data points.

In this work, only data from valves $\mathrm{A}$ for the working pressure of 5 bars are reported. Valve lift is reported in arbitrary units due to trade secret restrictions.

\subsection{Discharge valve (DV).}

Fig. $4-6$ show a rapid increase in the discharge duct, followed by a small decrease caused by an opening of a one-way valve to the storage tank. This is in agreement with the in-cylinder pressure which also rapidly rises and later shows a small decrease, and with the reed valve movement, which partially closes after first peak of valve lift, as apparent from the velocity profile shown in Fig. 7. When the pressures are matched, the reed valve opens and remains open during most of the discharge stroke. Only during the last phase the valve opening increases slightly. This is due to the pressure differences across the valve, during which the acceleration of the flow in a narrow slot leads to a pressure decrease and to an aerodynamic paradox [6]. 


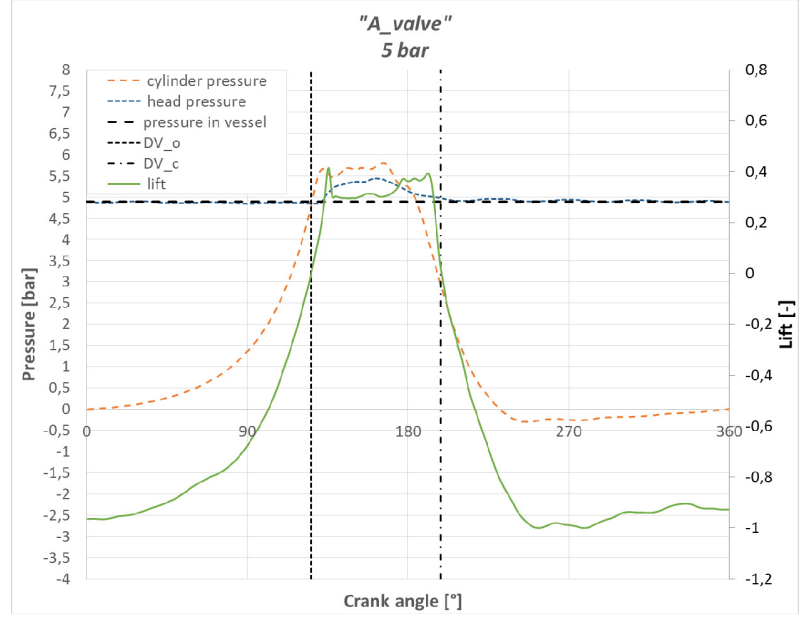

Figure 4. p-alfa diagram for $500 \mathrm{kPa}$ - discharge valve lift.

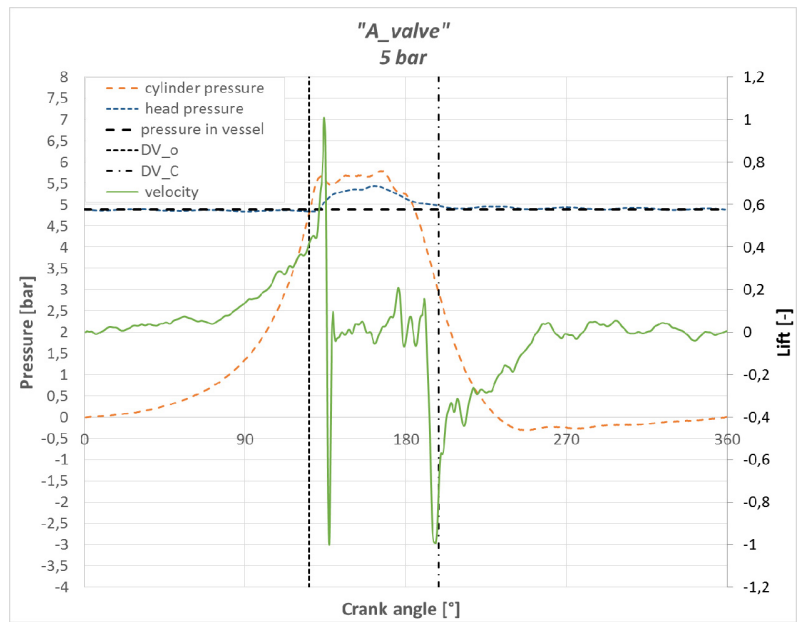

Figure 5. p-alfa diagram for $500 \mathrm{kPa}$ - discharge valve velocity.

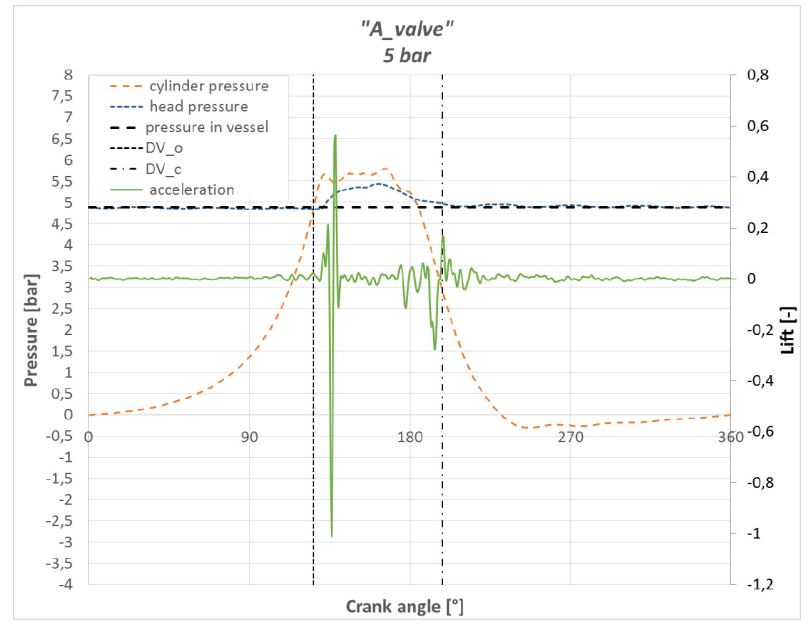

Figure 6. p-alfa diagram for $500 \mathrm{kPa}$ - discharge valve acceleration.

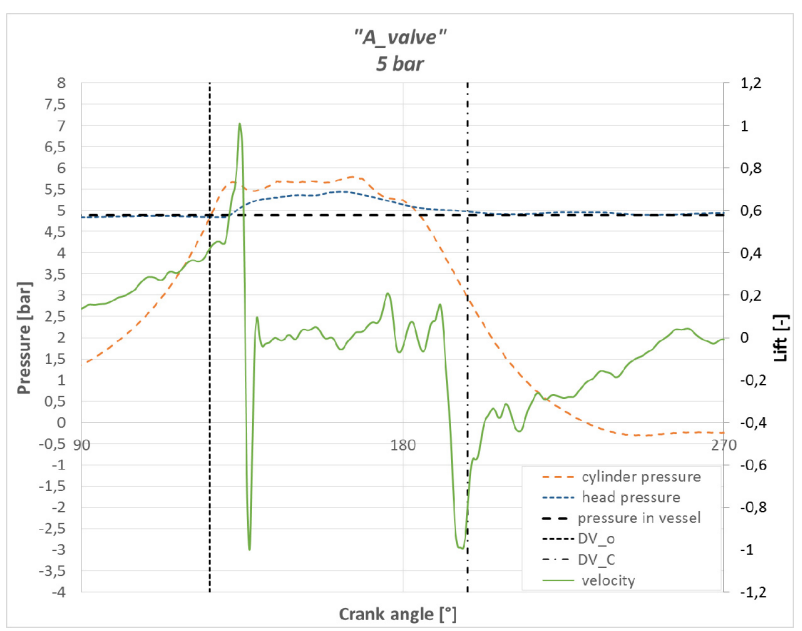

Figure 7. p-alfa diagram for 5 bar - detail of discharge valve velocity.

A numerical integration was performed on the velocity data to obtain the valve lift profile, using the boundary condition of zero lift $\mathrm{p}_{\text {cylindr }}=\mathrm{p}_{\text {head }}<0^{\circ}, \mathrm{CA} \mathrm{p}_{\text {cylindr max }}>$. This condition has ensured that the reference position is not skewed by the cylinder head overpressure. The acceleration of the valve was calculated as the numerical derivative of the velocity.

\subsection{Suction valve (SV).}

Fig. 8 - 10 show lift, velocity and acceleration of suction valve. A numerical integration was performed on the velocity data to obtain the valve lift profile. The integration constant could not be readily obtained using a boundary condition, as the suction valve is deformed by the pressure in the cylinder. Instead, zero lift was assigned to a time between crank angles of $0^{\circ}$ and $90^{\circ}$ during which:

1. there was a considerable decrease in valve acceleration (no major changes in acceleration),

2. there was at least a short period with a constant lift (no effect of in-cylinder pressure and valve flicker).

The zero lift position determined in this way was the compared with the lift at the beginning of the suction stroke and with acceleration following the suction valve opening, and corrected as necessary. The second point was not readily apparent, especially for valves „, $\mathrm{B}^{\text {“ }}$ and „D“, where flicker was leading into deformation caused by in-cylinder pressure. For these reasons, the actual beginning of the opening and end of the closing of the suction valve may differ by several crank angle degrees.

The results show that while using a vibrometer is seemingly simple and practical method with relatively easy analysis and interpretation of data, care should be taken to avoid creation of a measurement artefact originating out of the vibrations of the entire compressor unit, which in turn causes distortion and vibrations of the mounting of the vibrometer. The use of an optic fiber might be beneficial for resolving this problem. 


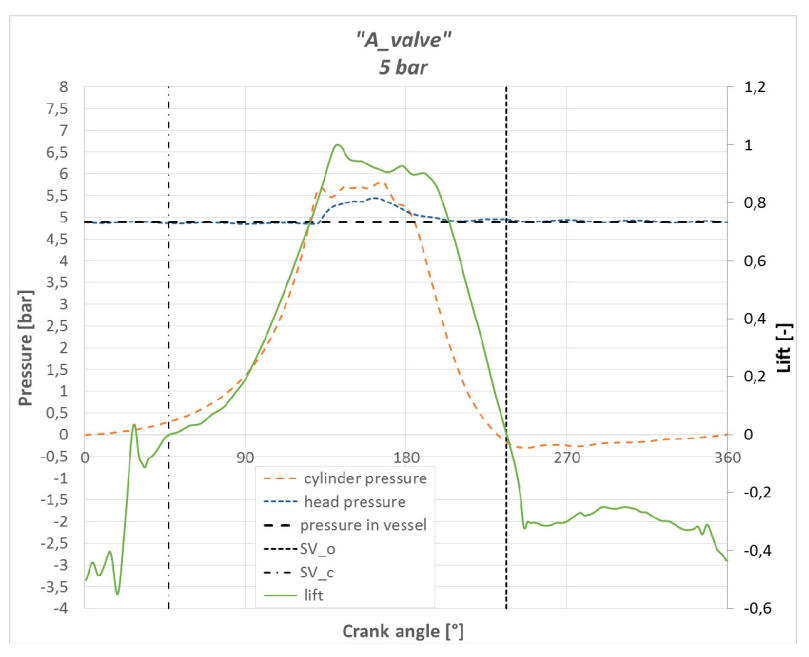

Figure 8. p-alfa diagram pro 5 bar - SV (lift).

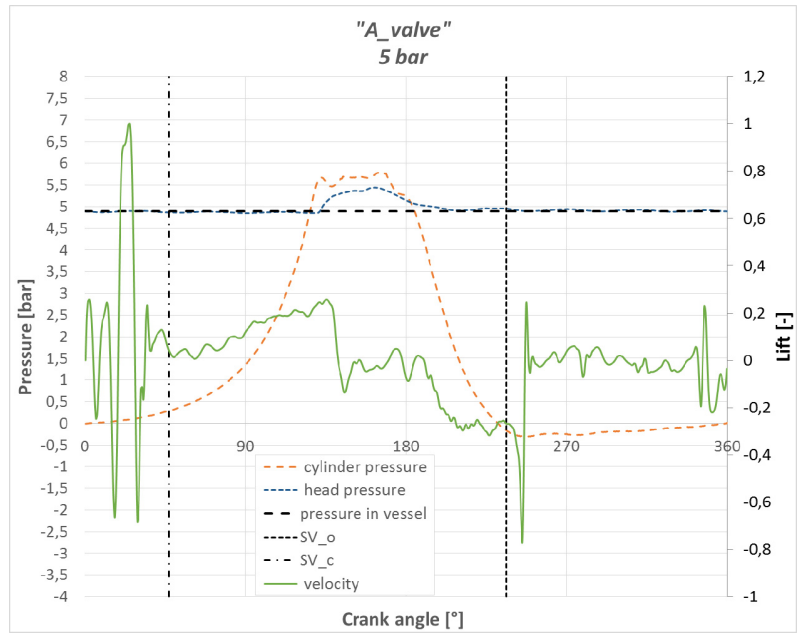

Figure 9. p-alfa diagram pro 5 bar - SV (velocity).

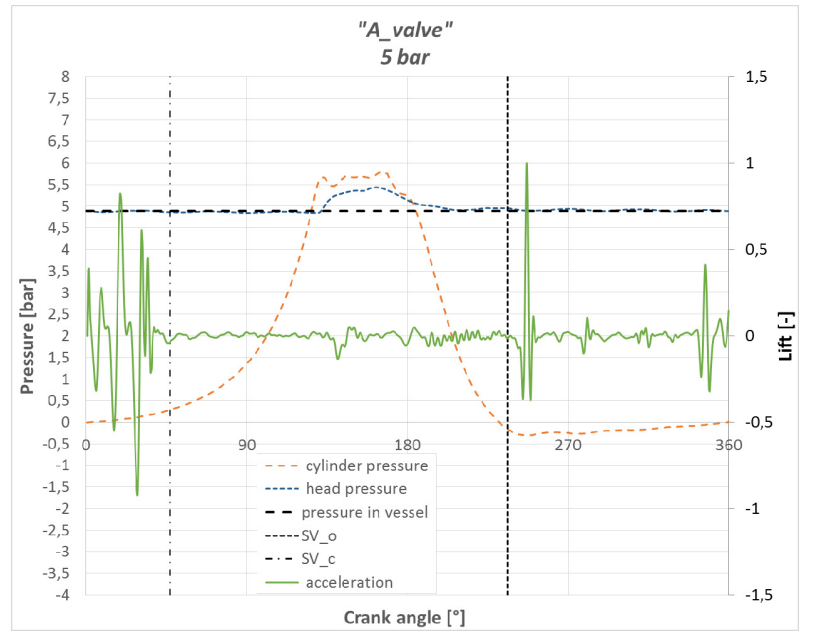

Figure 10. p-alfa diagram pro 5 bar - SV (acceleration).

\section{References}

1. A. Zimmermann, P. Hrnjak, "Visualization of the Opening Process of a Discharge Reed Valve in the Presence of Oil", International Compressor Engineering Conference, Paper 2369, (2014).
2. F. Lajus, C. Deschamps, M. Alves, "Numerical analysis of seat impact of reed type valves", 8th International Conference on Compressors and their Systems, London (2013)

3. Q. Tan, S. Pan, Z. Liu, X. Yu, Q. Feng, "Numerical study on transient effective flow and force areas of reed valve in a rotary compressor"; Proceedings of the Institution of Mechanical Engineers Part $A$ Journal of Power and Energy, 228(8), (2014)

4. A. Ludu, A. Beto, G. Regner, "Endoscope Video of Compressor Valve Motion and Pressure Measurement Assist Simulations for Design Improvements", International Compressor Engineering Conference., Paper 1420, (2000)

5. N. Paone, C. Santolini, E.P.Tomasini, "Application of a Laser Doppler Vibrometer to Evaluate Engine Poppet Valve Kinematics", $12^{\circ}$ Int.Modal Analysis Conf.,7, (1994)

6. W. Benenson, H. Stöcker, Handbook of physics. (2002) 